\title{
Diferencias entre los movimientos sacádicos, la discriminación auditiva y la lateralidad en niños de 7 a 11 años con y sin trastorno por déficit de atención con o sin hiperactividad
}

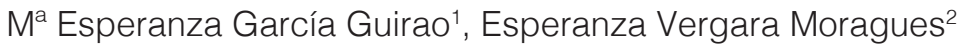 \\ Recibido: 27-04-2015 - Aceptado: 16-06-2015
}

\begin{abstract}
Resumen
El objetivo principal del trabajo es conocer si existen diferencias entre los movimientos sacádicos, la discriminación auditiva y la lateralidad en niños de 7 a 11 años con y sin TDA-H, así como la propuesta de un programa de intervención. Se valoró una muestra de 30 niños con edades entre los 7 y los 11 año; 15 presentaban TDA-H y los otros 15, no. Los movimientos sacádicos se evaluaron mediante el Test K-D; la discriminación auditiva mediante la evaluación del desarrollo fonológico (PAF) y la lateralidad, mediante el Test de Harris. Para el análisis estadístico se utilizó el análisis de relación cualitativo Chi-cuadrado. Los resultados confirmaron la existencia de diferencias significativas en los movimientos sacádicos y la discriminación auditiva entre los niños con y sin TDA-H, pudiéndose establecer que estas diferencias están relacionadas con el TDA-H, mientras que en lateralidad no se establecieron diferencias importantes entre ambos grupos.
\end{abstract}

Palabras clave: TDA-H, movimientos sacádicos, discriminación auditiva, lateralidad, programa de intervención.

\section{Differences between saccadic movements, auditory discrimination and laterality in children aged 7-11 years without attention deficit disorder with or without hyperactivity}

\begin{abstract}
The main objective of the study was to determine whether differences exist between saccades, auditory discrimination and laterality in children aged 7-11 years without ADHD, and the proposal of an intervention program. A sample of 30 children aged 7 to 11 years, 15 had ADHD and the other 15 was not assessed. Saccades were evaluated by Test KD, auditory discrimination by evaluating the phonological development (PAF) and laterality test by Harris. For statistical analysis, qualitative analysis regarding Chi - square
\end{abstract}

\footnotetext{
${ }^{1}$ Maestra de Educción Primaria, especialista en Neuropsicología y Educación. tarata86@gmail.com 2Investigadora principal del Grupo investigación Neuropsicología aplicada a la Educación y Profesora Adjunta de la Universidad internacional de la Rioja (UNIR). Doctora en Psicología. Máster en Neuropsicología. esperanza.vergara@unir.net
} 
test was used. The results confirmed the existence of significant differences in saccadic movements and auditory discrimination between children with and without ADHD, being able to establish that these differences are related to ADHD, whereas in laterality no significant differences were established between the two groups.

Keywords: ADHD, saccades, auditory discrimination, laterality intervention program.

\section{Introducción}

En la actualidad, el trastorno por déficit de atención con o sin hiperactividad (TDA-H, en adelante) es uno de los trastornos más comunes diagnosticados en las escuelas. Los estudios realizados hasta el momento muestran que alrededor de un $8 \%$ de la población infantil lo padece, llegando incluso a continuar en un $60 \%$ en la edad adulta (Gratch, 2000; Green y Chee, 2000; Orjales, 1998). Este diagnóstico, en ocasiones, se realiza de forma errónea dando lugar a que las personas que lo padecen desarrollen dificultades escolares, agresividad, tendencia a la violencia, ansiedad, depresión o problemas a la hora de relacionarse tanto con la familia como con los compañeros (Barkley, 1997; Montañés, 2008).

Es por ello que, el realizar un diagnóstico adecuado se convierte en primordial para poder atender correctamente a las necesidades de esta población, por lo que, en él, se debe incluir una evaluación de las variables neuropsicológicas que pueden repercutir en las personas que padecen el trastorno (por ejemplo, movimientos sacádicos, funcionalidad auditiva y lateralidad) (Zuluaga, 2011). Además, se tendrá que trabajar la estimulación del sistema nervioso central y la maduración de los diversos sistemas de procesamiento de la información y del control postural del niño, con el fin de lograr una reducción de los síntomas y una mejora a nivel escolar. Todo ello, a través del trabajo en diferentes áreas (ruta visual, auditiva, de movilidad y dietoterapia) con el objetivo de dar un enfoque global y multidisciplinar a la intervención de los niños que presentan TDA-H (López-Juez, 2008).

Actualmente, existen muchos trabajos relacionados con los procesos cognitivos como resultados del trastorno (atención, memoria, funciones ejecutivas...). Sin embargo, una de las preguntas de las que parte este trabajo es ¿cómo procesa la información el alumnado que presenta TDA-H? Por ello, una de las innovaciones del mismo es establecer las diferencias entre los movimientos sacádicos, la discriminación auditiva y la lateralidad en niños de 7 a 11 años con y sin TDA-H. Se llevó a cabo, a través de diferentes pruebas neuropsicológicas como el Test K-D para valorar los movimientos sacádicos, la prueba para la evaluación del desarrollo fonológico (PAF) para valorar la discriminación auditiva y el Test de Harris para evaluar la lateralidad de los niños de ambos grupos. Hasta lo que se conoce en la actualidad, no se había realizado aún una investigación en la que se establecieran las diferencias entre las variables mencionadas y el TDA-H, por lo que este trabajo aportará nuevas ideas que ayudarán considerablemente en la mejora de vida de estos sujetos.

El objetivo general en el que se basa el presente estudio es conocer si existen diferencias entre los movimientos sacádicos, la discriminación auditiva y la lateralidad en niños con edades comprendidas entre los 7 y 11 años con y sin TDA-H para que, una vez conocidas, se pueda proponer en un futuro un programa de intervención con el fin de resolver las dificultades que presenten.

En cuanto a los objetivos específicos son los siguientes: a) estudiar cuántos niños tienen dificultades en los movimientos sacádicos, la discriminación auditiva y en lateralidad cruzada y lateralidad homogénea y b) establecer las diferencias entre los problemas en movimientos sacádicos, discriminación auditiva y lateralidad de los niños con y sin TDA-H. 
BÚSQUEDA - Enero / Junio de 2015 - No. 14 (32 - 44)

\section{Metodología}

La pregunta de la cual se parte para dar comienzo al trabajo es, ¿los niños con TDA-H presentan una mayor dificultad en los movimientos sacádicos y discriminación auditiva y un mayor porcentaje de lateralidad cruzada que los niños que no padecen TDA-H?

\section{Objetivos e hipótesis}

En cuanto al objetivo general de este trabajo, ha sido conocer si existen diferencias entre los movimientos sacádicos, la discriminación auditiva y la lateralidad en niños con edades comprendidas entre 7 y 11 años con y $\sin$ TDA-H.

Los objetivos específicos son:

- Estudiar cuántos niños tienen problemas en movimientos sacádicos, discriminación auditiva y en lateralidad cruzada y lateralidad homogénea.

- Establecer las diferencias entre los problemas en movimientos sacádicos, discriminación auditiva y lateralidad de los niños con y sin TDA-H.

En cuanto a la hipótesis general del trabajo es que existen diferencias entre los movimientos sacádicos, la discriminación auditiva y la lateralidad de los niños de 7 a 11 años con y sin TDA-H.

Las hipótesis específicas son:

- Hipótesis 1: el alumnado con TDA-H presenta más problemas en los movimientos sacádicos.

- Hipótesis 2: el alumnado con TDA-H muestra más problemas en discriminación auditiva.

- Hipótesis 3: el alumnado con TDA-H presenta un mayor porcentaje de lateralidad cruzada.

\section{Diseño}

A la hora de realizar el trabajo se ha usado un diseño no experimental, cualitativo, descriptivo y correlacional. Se ha elegido este diseño porque tiene como base principal la investigaciónacción de un profesional en el aula y no hay una manipulación experimental de las variables, basándose principalmente en la observación del fenómeno dentro de un contexto específico para poder analizarse posteriormente. Además, es transversal porque se ha realizado la evaluación en un solo momento de tiempo.

\section{Población y muestra}

La población elegida para la realización del presente trabajo pertenece a varios centros. Los niños con TDA-H han sido escogidos de un centro específico para el TDA-H de la Comarca del noroeste murciano, mientras que los niños que no presentaban el trastorno han sido elegidos de un colegio concertado también de la misma ciudad. En cuanto a sus familias, se encuentran dentro de un nivel socioeconómico medio, que poseen características y niveles educativos similares.

En los criterios de inclusión se establecieron tener características similares y no estar inmerso en problemas escolares graves. Se excluyeron a aquellos alumnos con problemas graves de conducta y escolares, y a los que estuviesen bajo algún tratamiento farmacológico.

Teniendo en cuenta la tabla 2, la muestra se ha compuesto por 30 niños de edades comprendidas entre los 7 y los 11 años. La mitad de ellos presentaban TDA-H (diagnosticados a través de los criterios diagnósticos del DSM-V por profesionales expertos) y otra mitad, ni problemas escolares, ni de conducta. En relación con el sexo, tanto en el grupo de TDAH como el grupo sin TDAH, el 33,3\% eran niñas y el $66,7 \%$, niños. Por último, el $80 \%$ de ambos grupos estudiaba en Educación Primaria y el $20 \%$, en Educación Secundaria. Como se observa en la tabla, no existen diferencias entre estas variables, considerándose los grupos homogéneos para su comparación.

Variables de medida e instrumentos aplicados

En cuanto a las variables e instrumentos que se han aplicado, se establecen en la tabla 1, en la que se relaciona cada variable con el instrumento 
utilizado para evaluarla y las puntuaciones que se han usado.

\section{Variables}

Con el fin de trabajar, tanto los objetivos como las hipótesis establecidas anteriormente, se analizan diferentes variables como son los movimientos sacádicos, la discriminación auditiva, la lateralidad y presentar o no TDAH de la muestra elegida.

\section{Instrumentos}

\section{Movimientos sacádicos}

Para evaluar los movimientos sacádicos se ha usado el Test K-D (Evans, Richaman, Nicholson y Gaines, 1990), mediante el cual se han evaluado los seguimientos oculares, a través de una prueba de tipo visual durante la lectura. Se ha realizado leyendo números, con el fin de que no entre en juego la interpretación. Este test comprende varios subtes:
- El primero de ellos es de control, y se realiza para que el niño comprenda lo que se le está pidiendo, para lo cual se le presenta una carta con números unidos con marcas de seguimiento.

- El segundo test consiste en una carta con números, pero en este caso sin marcas de seguimiento.

- Y el último test, es el más complicado, ya que se le presenta una carta en la que los números están distribuidos sin muchas referencias.

A la hora de realizar los test, se les presentan las cartas a los sujetos y se les pide que las lean tan rápido y cuidadosamente como sea posible; se les toman el tiempo y anotan los errores y los resultados en una tabla. Para corregirlos, se utilizan las tablas en función de la edad. Además, deben leer un texto de su nivel escolar con el fin de medir el número de palabras que los niños leen por minuto.

Tabla 1. Variables, instrumentos y puntuaciones

\begin{tabular}{llll}
\hline \multicolumn{1}{c}{ Variable } & \multicolumn{1}{c}{ Instrumento } & Categorización cualitativa \\
\hline Movimientos sacádicos & $\begin{array}{l}\text { Test K-D para baremar los } \\
\text { movimientos sacádicos (1990). }\end{array}$ & $\begin{array}{l}\text { Ausencia de dificultad/presencia de } \\
\text { dificultad. }\end{array}$ \\
Discriminación auditiva & $\begin{array}{l}\text { Test de discriminación auditiva PAF } \\
\text { (Vallés, 1990). }\end{array}$ & $\begin{array}{l}\text { Ausencia de dificultad/presencia de } \\
\text { dificultad. }\end{array}$ \\
& Test de dominancia lateral de Harris & Homogénea/cruzada. \\
Lateralidad & (Harris, 1978). & \\
\hline
\end{tabular}

Fuente: Elaboración propia

Tabla 2. Análisis descriptivo de la muestra

\begin{tabular}{lrrr}
\hline & \multicolumn{1}{c}{ TOTAL } & \multicolumn{1}{c}{ TDAH } & No TDAH \\
\hline $\begin{array}{l}\text { Edad } \\
\text { (Media/desviación tipo) }\end{array}$ & $8.77(1.45)$ & $8.77(1.45)$ & $8.77(1.45)$ \\
Sexo & & & \\
Niña & $10(33.4)$ & $5(33.3)$ & $5(33.3)$ \\
Niño & $20(66.7)$ & $10(66.7)$ & $10(66.7)$ \\
Curso escolar & & & \\
Primaria & $24(80)$ & $12(80)$ & $12(80)$ \\
Secundaria & $6(20)$ & $3(20)$ & $3(20)$ \\
\hline
\end{tabular}

Fuente: Elaboración propia 
BÚSQUEDA - Enero / Junio de 2015 - No. 14 (32 - 44)

Por último, se realizó una prueba de seguimientos oculares, en la que el niño debió seguir la punta del lápiz sin realizar ningún movimiento de otra parte del cuerpo. El ojo tendrá que realizar un movimiento tan suave como el lápiz. Se situó a $40 \mathrm{~cm}$ de la cara y se desplazó en horizontal y vertical. Esta prueba ofrece valores normalizados para las diferentes edades. Estos valores pueden servir como indicadores para valorar la presencia de dificultad.

\section{Discriminación auditiva}

Para evaluar la discriminación auditiva se ha pasado la prueba de discriminación auditiva PAF (Vallés, 1990) que ha consistió en leer a cada uno de los sujetos unas palabras, que repitieron posteriormente. Es importante que los alumnos estuviesen colocados de espaldas a nosotros para que no vean cómo se vocaliza y solamente escuchen la palabra. La existencia de errores en la prueba a partir de los 6 años indica la presencia de dificultades de discriminación auditiva.

\section{Lateralidad}

Para evaluar la lateralidad, se ha utilizado el Test de dominancia lateral de Harris (Harris, 1978), que es un manual constituido por varios test recopilados y elaborados por el autor, como consecuencia de los estudios que realizó sobre la lateralidad. Es de gran utilidad a la hora de evaluar a los sujetos que tienen problemas en la lectura, la ortografía y la escritura. Estudia, por tanto, la dominancia lateral (ojo, mano, pie). Se aplica individualmente, a partir de los 6 años de edad. Algunas de las actividades que establece este test son las siguientes:

- Preferencia de la mano: Lanzar una pelota, dar cuerda a un reloj, golpear con un martillo, cepillarse los dientes, etc.

- Dominio de ojos:

Mirar por un agujero de un cartón, mirar por un telescopio o mirar por un calidoscopio.

- Dominio de pies:

Patear una pelota, conducir una pelota o meter un gol.
Así pues, tras obtener los diferentes niveles de las variables analizadas, se podrán establecer tanto los resultados como los análisis de los mismos, en relación con las hipótesis planteadas y que serán claves a la hora de proponer el programa de intervención.

\section{Procedimiento}

Los pasos que se dieron a la hora de llevar a cabo el estudio fueron los siguientes. En primer lugar, se contactó con los directores de los centros con los que se iba a pasar las pruebas y se les explicó el objetivo del mismo, la muestra de alumnos que se iba a necesitar y el tiempo necesario para la evaluación de cada sujeto. En segundo lugar, se les solicitó permiso para la realización de las pruebas, encargándose los directores de cada centro de informar tanto a los padres como a los profesores de los niños. Tras la obtención del permiso, se eligió la muestra de 30 niños: 15 de un centro específico de TDA-H y otros 15, de un colegio concertado con cocientes intelectuales similares.

Las pruebas se aplicaron durante la mañana a los niños del centro concertado y durante la tarde a los niños del centro de TDA-H, en sesiones dé 45 minutos para cada niño, en las cuales se pasó primero la prueba de movimientos sacádicos, después, la de discriminación auditiva y, por último, las de lateralidad. Las pruebas fueron aplicadas tanto por los orientadores del centro como por una persona experta en Neuropsicología y Educación, de tal forma que los niños salían a un aula cedida por cada centro, luminosa y tranquila, para la realización de las pruebas.

\section{Análisis de datos}

En primer lugar, se realizó un análisis de datos de tipo descriptivo en relación con la muestra elegida y a las variables. En segundo lugar, se llevó a cabo un análisis de relación de las hipótesis planteadas mediante el análisis de Chicuadrado. Para el tratamiento estadístico de los datos se utilizó el programa Excel y el programa SPSS 15.0 para Windows (complemento de Excel). 


\section{Resultados}

Como se pudo observar en la tabla 2, se presetan los resultados a partir del análisis descriptivo de las variables del estudio y posteriormente, se establecen los resultados del análisis de relación de las variables del estudio.

Objetivo 1. Análisis descriptivo de las variables del estudio

A continuación, en cada una de las tablas de este apartado, se recogen los resultados en relación con el análisis descriptivo de las variables de estudio: movimientos sacádicos, discriminación auditiva y lateralidad, de tal forma que:

En el análisis descriptivo de los movimientos sacádicos, tabla 3 , se observa que hay más niños con TDA-H que presentan dificultad, más concretamente un $73.3 \%$ de ellos; mientras que hay más niños sin TDA-H que no presentan ninguna dificultad en movimientos sacádicos, más concretamente un $86.7 \%$.
En el análisis descriptivo de la discriminación auditiva, tabla 4, se observa que al igual que en los movimientos sacádicos, hay más niños con presencia de dificultad en discriminación auditiva que presentan TDA-H (53.3\%); mientras que sin dificultad, son mayoría los niños que no tienen TDA-H (86.7\%).

En cuanto al análisis descriptivo de la lateralidad, tabla 5, se observa como dentro del grupo con presencia de dificultad, son mayoría los niños que tienen TDA-H (46.7\%); y dentro del grupo con ausencia de dificultad, el 73.3\%.

Es importante destacar, que a la hora de categorizar las variables de cara a los análisis estadísticos, el grupo de alumnos quedó dividido en lateralidad homogénea y lateralidad cruzada.

Objetivo 2. Análisis de relación de las variables del estudio

En el presente apartado, se establecen los resultados con base en el análisis de relación de las variables del estudio: movimientos sacádicos, discriminación auditiva y lateralidad.

Tabla 3. Análisis descriptivo de los movimientos sacádicos

\begin{tabular}{|c|c|c|c|c|}
\hline & & Grupos & $\mathbf{N}$ & Porcentaje (\%) \\
\hline \multirow{4}{*}{$\begin{array}{l}\text { Movimientos } \\
\text { sacádicos }\end{array}$} & Presencia de dificultad & Con TDA-H & 11 & 73.3 \\
\hline & & Sin TDA-H & 2 & 13.3 \\
\hline & Ausencia de dificultad & Con TDA-H & 4 & 26.7 \\
\hline & & Sin TDA-H & 13 & 86.7 \\
\hline
\end{tabular}

Fuente: Elaboración propia

Tabla 4. Análisis descriptivo de la discriminación auditiva

\begin{tabular}{lcccc}
\hline & Grupos & N & Porcentaje (\%) \\
\hline \multirow{3}{*}{$\begin{array}{l}\text { Discriminación } \\
\text { auditiva }\end{array}$} & Presencia de dificultad & Con TDA-H & 8 & 53.3 \\
& & Sin TDA-H & 2 & 13.3 \\
& Ausencia de dificultad & Con TDA-H & 7 & \\
& & Sin TDA-H & 13 & 46.7 \\
& & & & 86.7 \\
\hline
\end{tabular}

Fuente: Elaboración propia 
BÚSQUEDA - Enero / Junio de 2015 - No. 14 (32 - 44)

Tabla 5. Análisis descriptivo de los resultados para la variable lateralidad por cada uno de los grupos evaluados

\begin{tabular}{lllll}
\hline Grupos & \multicolumn{1}{c}{ Análisis } & N & Porcentaje (\%) \\
\hline & Con TDA-H & Diestro, cruce visual izquierdo & 3 & \\
& & Diestro, cruce audición izquierdo & 3 & 46.7 \\
& Cruzada & 1 & \\
Lateralidad & Sin TDA-H & Diestro, cruce manual izquierdo & 1 & 26.7 \\
& & Diestro, cruce audición izquierdo & 2 & \\
& Cruzada & 1 & 53.3 \\
& Con TDA-H & Zurdos & 5 & \\
& Diestros & 2 & 73.3 \\
& Sin TDA-H & Zurdos & 3 & \\
& Diestros & 8 & \\
\hline
\end{tabular}

Fuente: Elaboración propia

Tabla 6. Análisis descriptivo de la lateralidad

\begin{tabular}{lcccc}
\hline & Grupos & N & Porcentaje (\%) \\
\hline \multirow{3}{*}{ Lateralidad } & Cruzada & Con TDA-H & 7 & 46.7 \\
& & Sin TDA-H & 4 & 26.7 \\
& & & 8 & \\
& Homogénea & Con TDA-H & 11 & 53.3 \\
& & Sin TDA-H & 73.3 \\
\hline
\end{tabular}

Fuente: Elaboración propia

En relación con los movimientos sacádicos, tabla 7 , podemos observar cómo el valor de la $p$ es menor de 0.05, por lo que podemos decir que, en relación con esta variable, sí hay diferencias entre los niños con TDA-H y los niños sin el trastorno, presentando más dificultades aquellos niños que lo padecen, cumpliéndose así la hipótesis número uno.

En relación con la discriminación auditiva, tabla 8 , el valor de p sigue siendo inferior a 0.05 , por lo que, al igual que en el caso anterior, se cumple la hipótesis número dos, es decir, sí hay diferencias en discriminación auditiva entre niños con TDA-H y sin TDA-H, siendo los niños con el trastorno, quienes evidencian más dificultades.

En el caso de la lateralidad, tabla 9, el valor de la p es superior a 0.05 , por lo que, en este caso, no se cumple la hipótesis establecida anteriormente, ya que en relación con la lateralidad, los niños con TDA-H no muestran más dificultades en esta variable que los niños $\sin \mathrm{TDA}-\mathrm{H}$. 
Ma Esperanza García G., Esperanza Vergara M. - Diferencias entre los movimientos sacádicos

Tabla 7. Análisis de relación de los movimientos sacádicos

\begin{tabular}{lcccc}
\hline \multirow{2}{*}{$\begin{array}{l}\text { Movimientos } \\
\text { sacádicos }\end{array}$} & \multicolumn{4}{c}{ TDA-H } \\
\cline { 2 - 4 } & \multicolumn{2}{c}{ Total N (\%) } & Chi-cuadrado & Pusencia \\
\cline { 2 - 5 } & $4(26.7 \%)$ & $13(86.7 \%)$ & 10.995 &, 001 \\
Presencia & & & \\
\hline
\end{tabular}

$p<0.05$ hay diferencias / $p>0.05$ no hay diferencias

Fuente: Elaboración propia

Tabla 8. Análisis de relación de la discriminación auditiva

\begin{tabular}{|c|c|c|c|c|}
\hline \multirow{3}{*}{$\begin{array}{l}\text { Discriminación } \\
\text { auditiva }\end{array}$} & \multicolumn{4}{|c|}{ TDA-H } \\
\hline & \multicolumn{2}{|c|}{ Total N (\%) } & \multirow[b]{2}{*}{ Chi-cuadrado } & \multirow[b]{2}{*}{$\mathbf{P}$} \\
\hline & Presencia & Ausencia & & \\
\hline \multirow[t]{2}{*}{ Ausencia de dificultad } & $7(46.7 \%)$ & $13(86.7 \%)$ & & \\
\hline & & & 5.400 & ,020 \\
\hline Presencia de dificultad & $8(53.3 \%)$ & $2(13.3 \%)$ & & \\
\hline
\end{tabular}

Tabla 9. Análisis de relación de la lateralidad

\begin{tabular}{|c|c|c|c|c|}
\hline \multirow{3}{*}{ Lateralidad } & \multicolumn{4}{|c|}{ TDA-H } \\
\hline & \multicolumn{2}{|c|}{ Total N (\%) } & \multirow[b]{2}{*}{ Chi-cuadrado } & \multirow[b]{2}{*}{$\mathbf{P}$} \\
\hline & Presencia & Ausencia & & \\
\hline \multirow[t]{2}{*}{ Definida } & $8(53.3 \%)$ & $11(73.3 \%)$ & & \\
\hline & & & 1.292 & ,256 \\
\hline No definida & $7(46.7 \%)$ & $4(26.7 \%)$ & & \\
\hline
\end{tabular}

$p<0.05$ hay diferencias / $p>0.05$ no hay diferencias

Fuente: Elaboración propia

\section{Discusión y conclusiones}

Dada la importancia de las variables analizadas en la investigación, el objetivo que se ha perseguido con la realización de este trabajo ha sido conocer si existen diferencias entre movimientos sacádicos, discriminación auditiva y lateralidad en niños con edades comprendidas entre 7 y 11 años con y sin TDA-H. Todo ello, con el fin de proponer un programa de intervención para resolver las dificultades que presenten los niños.

En cuanto a los resultados obtenidos en las diferentes pruebas en relación con las hipótesis planteadas, se han establecido las diferencias existentes en las diversas variables estudiadas entre niños de 7 a 11 años con y sin TDA-H, 
BÚSQUEDA - Enero / Junio de 2015 - No. 14 (32 - 44)

menos en lateralidad que los resultados fueron diferentes. A continuación, se establecen las conclusiones a partir de los resultados obtenidos, así como su relación con las hipótesis planteadas.

Teniendo en cuenta la hipótesis 1, sí existen diferencias significativas en los movimientos oculares entre los niños con y sin TDA-H. Tras la realización de las pruebas y el establecimiento de los resultados, se puede confirmar la primera hipótesis, por lo que se puede decir que esta es una variable a tener en cuenta a la hora de trabajar con los niños que padecen el trastorno.

Así, Vergara (2008) determina que el desarrollo de la ruta visual en los niños forma parte de un proceso muy complejo, que no depende solamente de tener buena agudeza visual, sino que en este proceso, el cerebro tiene que desarrollar la capacidad de aprender a interiorizar los estímulos visuales, al mismo tiempo que se produce la maduración del Sistema Nervioso Central (Ferré, 2002), lo cual se convierte en esencial para que los niños con dificultades en esta ruta puedan mejorar la lectura, para entender lo que leen e interiorizar las acciones.

Por otra parte, la hipótesis 2 establece que sí existen diferencias significativas en la discriminación auditiva entre los niños de 7 a 11 años con y sin TDA-H. Tras la aplicación de las diferentes pruebas, así como el establecimiento de los resultados, se puede confirmar la segunda hipótesis, por lo que se puede decir que esta es una variable a tener en cuenta a la hora de trabajar con los niños afectados por el trastorno, al igual que la anterior.

Así pues, teniendo en cuenta investigaciones como la de Mercugliano (1999) que apoyan esta hipótesis, Ios niños que presentan TDA-H tienen problemas a la hora de procesar la información auditiva, por lo que, en muchas ocasiones parece no oyesen. Esto se produce porque experimentan algún problema a la hora de procesar y discriminar la información auditiva. Todo ello, hace que el niño desarrolle un bajo rendimiento escolar, ya que para que el lóbulo frontal pueda procesar la información de forma adecuada es necesario que los receptores auditivos funcionen manera correcta (López-Juez, 2004).

Finalmente, según la hipótesis 3, sí hay diferencias significativas en lateralidad entre niños de 7 a 11 años con y sin TDA-H. Tras el análisis de los resultados obtenidos en las pruebas, se puede determinar que, en relación con lateralidad, no existen diferencias significativas en la muestra elegida; esto podría deberse, entre otras cuestiones, a que la muestra ha sido muy pequeña.

En este sentido, según diversos autores como Ferré Veciana (2002), hay ocasiones en las que los problemas en la lateralidad pueden desembocar en dificultades en hiperactividad y atención, pero pueden existir otras causas; por lo que, para mejorar el desarrollo lateral de estos niños, este autor, propone la terapia sensorial, neurológica y psicomotriz.

En general, los resultados obtenidos apoyan las investigaciones previas (menos en lateralidad), que señalan la importancia de estimular al sistema nervioso central con el fin de que los diferentes sistemas de procesamiento de la información y de control postural de los sujetos maduren y así, conseguir la mejora del rendimiento escolar como consecuencia de la reducción de la sintomatología. Todo ello, a través del trabajo en diferentes áreas (ruta visual, auditiva, de movilidad y dietoterapia), con la finalidad de desarrollar una intervención con los niños que presentan TDA-H basada en un enfoque global y multidisciplinar (López-Juez, 2008).

Por lo tanto y teniendo en cuenta todo lo establecido anteriormente, las conclusiones de este trabajo se pueden resumir en las siguientes:

- Los movimientos sacádicos es una variable que influye en los niños que tienen TDA-H, ya que existen diferencias significativas entre los niños con y sin el trastorno, según los resultados obtenidos tras la realización de las pruebas, por lo que a la hora de 
Mª Esperanza García G., Esperanza Vergara M. - Diferencias entre los movimientos sacádicos

trabajar con estos niños deberá tener en cuenta esta variable. El realizar actividades dirigidas a ellos que exhiban dificultad en esta variable, independientemente de si tienen o no TDA-H; se puede mejorar o realizar nuevas actividades en función de las características y necesidades de ellos en cada momento. Un ejemplo de actividad que se podría desarrollar para esta variable sería la siguiente: seguir con los ojos los movimientos de una pelota de tenis colgada del techo o los movimientos de la luz de una linterna, entre otras.

- La discriminación auditiva, al igual que los movimientos sacádicos, son una variable que suele presentarse en los niños con TDA-H. Esto se apoya en los resultados obtenidos que establecen las diferencias en la discriminación auditiva entre los niños con y sin el trastorno, por lo que, del mismo modo que la anterior, esta deberá ser tenida en cuenta al realizar actividades, como por ejemplo, al utilizar el ordenador, los alumnos realizarán una actividad en la que deberán agrupar los sonidos que suenen igual; o los niños durante unos minutos tendrán que estar en silencio, cerrar los ojos y decir qué sonidos escuchan a su alrededor.

- En cuanto a la lateralidad, las pruebas realizadas no establecen diferencias significativas entre los niños con y sin TDA-H. En cualquier caso, sería interesante seguir trabajando en esta línea de investigación para conocer, con mayor profundidad, el papel de la lateralidad en el rendimiento académico.

\section{Limitaciones}

Tras la realización del trabajo, se puede establecer la presencia de varias limitaciones, así como algunas maneras de paliarlas para investigaciones futuras.

En relación con la muestra elegida, esta es muy pequeña, ya que se ha compuesto de 30 niños, de los cuales solamente 15 tenían TDA-H, con lo cual se limitan las posibilidades de su generalización al resto de la población. Sería conveniente, por tanto, utilizar una muestra más elevada para poder seguir teniendo datos más robustos en este campo. En esta línea, las edades de los participantes se han limitado a ser de 7 a 11 años, con lo cual los resultados no pueden ser generalizados a niños de otras edades. En este caso, sería interesante ampliar la edad de la muestra y hacer varios grupos, primaria y secundaria, para ver cómo afecta el trastorno a lo largo de la infancia y adolescencia de los sujetos.

Por otra parte, se podrían haber utilizado pruebas de discriminación visual en las que no solo se evalúen variables como los movimientos sacádicos, sino también otras como la acomodación o la convergencia.

Finalmente, una limitación importante se refiere tanto al tiempo de aplicación de las pruebas como al horario de aplicación a los niños con TDA-H. En cuanto al tiempo de aplicación ha sido muy escaso y los responsables de los distintos centros pusieron muchas objeciones a la hora de realizar las pruebas. Además, solo se les aplicaran las pruebas a los niños con TDA-H en horario de tarde, dado que solo estan en el centro en este intervalo de tiempo. En próximas investigaciones sería conveniente que el horario de aplicación de las pruebas fuera en ambos horarios y grupos.

A pesar de las limitaciones descritas, los resultados obtenidos tras la realización del trabajo han sido muy importantes, debido a que han aportado un primer estudio exploratorio de la relación de los movimientos sacádicos, la discriminación auditiva y la lateralidad en relación con el TDA-H. Desde este punto de vista, el trabajo establece la importancia de tener en cuenta las variables anteriores a la hora de tratar a los niños con TDA-H. Los resultados obtenidos se podrían usar en futuras investigaciones llevadas a cabo tanto por profesores, psicólogos clínicos y escolares, con el fin de profundizar y avanzar más en la investigación. De todos modos, este tema tendría que formar parte de un análisis más profundo en investigaciones futuras. 
BÚSQUEDA - Enero / Junio de 2015 - No. 14 (32 - 44)

\section{Prospectiva}

A partir de las averiguaciones de este estudio, se pone de manifiesto la importancia de actuar sobre las variables neuropsicológicas que influyen en el TDA-H, con el objetivo de comprender mejor a los niños que padecen el trastorno, así como ayudarles a mejorar su rendimiento escolar.

Por todo ello, sería interesante volver a realizar la investigación, en este caso, a través de un aumento tanto de la muestra elegida como de los instrumentos de evaluación de las variables neuropsicológicas implicadas en el estudio, con la finalidad de profundizar más en su relación con el TDA-H y con los déficit que los niños con el trastorno sufren en relación con el rendimiento escolar. Así pues, en futuras investigaciones se podrían utilizar, además de los que ya se han usado, otros instrumentos de medida con el fin de reforzar los resultados obtenidos. Se podrían extrapolar las pruebas a niños de otras edades, es decir, aumentar el rango de edad a niños de educación secundaria obligatoria, con el fin de que los resultados obtenidos puedan considerarse como generales y, por lo tanto, sea posible extenderse a toda la población escolar.

Finalmente, es factible abrir futuras investigaciones que se centrasen en diferentes variables neuropsicológicas. Estas variables podrían ser, entre otras, la convergencia, la acomodación, la atención, la memoria o las relaciones con su entrono más cercano, con el objetivo de establecer cómo influyen estas en los sujetos que tienen TDA-H, cuáles son las diferencias en relación con los sujetos que no lo padecen y qué programas de intervención se podrían proponer en función a los resultados obtenidos.

\section{Referencias bibliográficas}

\author{
Álvarez, L. (2007). Desarrollo de los procesos \\ atencionales mediante actividades \\ adaptadas. Papeles del psicólogo, 28, \\ 211-217.
}

American Psycological Association (2014). DSM-V. Publication of the American Psycological Association. Washington, D.C.

Arco, J. L., Fernández, F. D. y Hinojo, F. J. (2004). Trastorno por déficit de atención con hiperactividad. Psicothema, 16 (3), 408-414.

Artigas-Pallarés, J. (2003). El trastorno del déficit de atención con o sin hiperactividad en la consulta del pediatra. Revista pediatría de atención primaria.

Artigas-Pallarés, J. y Narbona, J. (2011). Trastornos del neurodesarrollo. Barcelona: Viguera Editores.

Barkley, R. A. (1997). Behavioral inhibition, sustained attention, and executive functions: Constructing a inifyling theory of ADHD. Psychological Bullentin, 121: 65-94.

Barkley, R. A. (1998). Attention-Déficit Hyperactivity Disorder. México: Guilford.

Barkley, R. A., Copeland y Sivage (1980). A self control classroom for hyperactive children. Journal of Autism and Developmental Disorders, 1: 75-89.

Bloomerg, H. (2008). Attention deficit disorder and hyperactivity and its development. México: Guilford.

Castellanos, F. X. (2002). Developmental trajectories of brain volumen abnomalities in children and adolescents with attention deficit/hyperactivity disorder. The Journal of the American Medical Association, 288, 1740-1748.

Corral, A. y Pardo de León, P. (2001). Psicología evolutiva I. Madrid, España: Universidad nacional a distancia.

Dougch (1985). Diccionario de psicología. Barcelona: Hender. 
Mª Esperanza García G., Esperanza Vergara M. - Diferencias entre los movimientos sacádicos

Estalayo, V. (2005). Inteligencia auditiva: técnicas de estimulación prenatal infantil y permanente. Madrid: Biblioteca nueva.

Evans, B., Richaman, J.E, Nicholson, S. B, y Gaines C. S. (1990). A new visual verbal Saccade Test. The developmental Eye Movement test (K-D). J Am Optom Assoc. 61:134.134.

Ferré, J. (2002). Trastornos de la atención y la hiperactividad. Diagnóstico. Madrid: Ediciones Lebrón.

Ferré, J. y Ferré, M. (2010). Déficit de atención e hiperactividad. 100 preguntas. 100 respuestas. Barcelona: Lebrón.

Ferré, J., y Irabau, E. (2002). El desarrollo neurofuncional del niño y sus trastornos. Madrid: Lebón.

Gioia, G.A., Isquith, P.K., Guy, S.C. y Kenworthy, L. (2000). Behavior Rating Inventory of Executive Function. Psychological Assessment Resources. Florida: Lutz.

Gladys, A. (2013). Problemas de aprendizaje: discriminación auditiva. Madrid: Universidad nacional de educación.

Gratch, L. (2000). El trastorno por déficit de atención. Buenos Aires: Panamericana.

Green, C.I. y Chee, K. (2000). El niño muy movido o despistado. Buenos Aires: Panamericana.

Groenewegen, H. L. y Uylings, H. B. M. (2000). Cognition, emotion and autonomic responses: The integrative role of the prefrontal cortex and limbic structures. Progress in Brain Research, 126, 3-28.

Harris, A. J. (1978). Test de dominancia lateral de Harris. Madrid: TEA.

Houghton, S. (1999). Differential patterns of executive function in children with Attention Deficit Hyperactivity Disorder according to gender and subtype. Journal of Child Neurology, 14, 801-805.

Kandel, E. R. (1991). Principles of Neural Science. New York: McGraw.

López-Campo, G. X. (2004). Componentes de las pruebas de memoria y función visoconstruccional en DDA. Tesis de Maestría en Neuropsicología no publicada. Medellín: Universidad de San Buenaventura.

López-Juez, M. (2008). ¿Por qué yo no puedo? Bases biológicas de los problemas de aprendizaje. Madrid: Neocortex.

Mercugliano, M. (1999). What is attention deficit/ hyperactivity disorder? PediatrClin North. Am, 46(5):831-43.

Montañés, F. (2008). Trastorno por Déficit de Atención con Hiperactividad (TDAH). Diario médico.

Mulas, F. (2006). TDAH preescolar: Diagnóstico e intervención terapéutica. Valencia, España: XIV Curso Internacional de Actualización en Neuropediatría y Neuropsicología Infantil.

Orjales, I. y Polaino-Lorente, A. (2001). Programas de Intervención Cognitivo-conductual para niños con Déficit de Atención con Hiperactividad. Madrid: CEPE.

Orjales, V.I. (1998). Déficit de Atención con Hiperactividad. Madrid: CEPE.

Rief, S. (1999). Cómo tratar y enseñar al niño con problemas de atención e hiperactividad. Técnicas, estrategias e intervenciones para el tratamiento de niños con TDA/ TDAH. Madrid: Paidós.

Rodríguez, C., González-Cesto, P., Álvarez, D. y Fernández, M. (2012). Neuropsycoloical analysis of the difficulties in dislexia throug sensory fusión. International Journal of clinical and health psychology, 12, 69-80. 
BÚSQUEDA - Enero / Junio de 2015 - No. 14 (32 - 44)

Shallice, T. (2002). Executive function profile of children with attention deficit hyperactivity disorder. Development Neuropsychology, 21, 43-71.

Vallés, A. (1990). Prueba de discriminación auditiva PAF. Madrid: CEPE.

Vergara, C. (2008). El aprendizaje escolar y sus dificultades. Una lectura psicoanalítica. Revista Electrónica de Psicología Social. Poiésis, (16), 01-07.
Zuluaga, J. B. (2011). Evolución en la atención, los estilos cognitivos y el control de la hiperactividad en niños, niñas con diagnóstico de trastorno deficitario de atención con hiperactividad (TDAH), a través de una intervención sobre la atención. Colombia Internacional Journal of Psycological Research. 